\title{
Colletotrichum nymphaeae var. entomophilum var. nov. a natural enemy of the citrus scale insect, Praelongorthezia praelonga (Hemiptera: Ortheziidae)
}

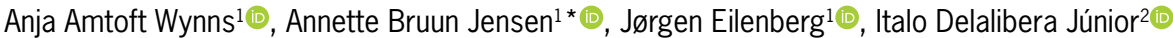

\author{
${ }^{1}$ University of Copenhagen - Dept. of Plant and \\ Environmental Sciences, Thorvaldsensvej 40, 1871 - \\ Frederiksberg C - Denmark. \\ 2Universidade de São Paulo/ESALQ - Depto. de Entomologia \\ e Acarologia, Av. Pádua Dias, 11 - 13418-900 - Piracicaba, \\ SP - Brasil. \\ *Corresponding author <abj@plen.ku.dk>
}

Edited by: Richard V. Glatz

Received August 20, 2018

Accepted April 30, 2019

\begin{abstract}
The citrus scale insect Praelongorthezia praelonga (Douglas), a major pest of citrus and other economically important crops, has only two commonly documented natural enemies: an entomopathogenic strain of the fungus Colletotrichum nymphaeae (Pass.) Aa and several parasitoids. The entomopathogenic strain of $C$. nymphaeae, formerly recognized under the synonym C. gloeosporioides $\mathrm{f}$. sp. ortheziidae, is under development for commercial application as a biological control agent in citrus in Brazil-the top exporter of citrus globally. The synonomy of C. gloeosporioides $\mathrm{f}$. $\mathrm{sp}$. ortheziidae with $\mathrm{C}$. nymphaeae remains based on limited DNA sequence data and without morphological study. To qualify for future approval as a biological control agent by federal agencies in Brazil and the European Union, the circumscription of a microorganism must be explicit and without ambiguities. Herein, through morphological study and phylogenetic analysis of five DNA regions we clarify the circumscription and affinity of entomopathogenic $C$. nymphaeae and describe it as a new variety.
\end{abstract}

Keywords: Biological control, Colletotrichum (Sordariomycetes: Glomerellaceae), entomopathogen

\section{Introduction}

Colletotrichum Corda (Ascomycota: Sordiariomycetes: Glomerallaceae) is a cosmopolitan and speciose genus of fungi comprised largely of plant symbionts (Damm et al., 2012; Hanlin, 1998). The host range within the genus is broad with both pathogenic and endophytic species reported from all major lineages of land plants (MacKenzie et al., 2009; Manamgoda et al., 2013; Photita et al., 2005). Two Colletotrichum taxa are entomopathogenic: C. fiorinae and C. nymphaeae. Colletotrichum fiorinae was described from the hemlock scale insect Fiorna externa Ferris; however, $C$. nymphaeae is a widespread plant pathogen in which entomopathogencity is an exception and limited to strains isolated from the citrus scale insect Praelongorthezia praelonga (Douglas) (syn. Orthezia praelonga Douglas) (Hemiptera: Ortheziidae) (Marcelino et al., 2008).

Entomopathogenic $C$. nymphaeae is of particular interest because the citrus scale insect (P. praelonga), its primary host, is a serious pest of Citrus spp. and other major economic plants such as coffee, figs and ornamentals (Garcia-Roa, 1995). Citrus scale is considered a pest wherever it is found because of its elevated reproductive rate and highly polyphagous nature (Kondo et al., 2013). The regularly occurring natural enemies of citrus scale are currently limited to $C$. nymphaeae and several parasitoids (Kondo et al., 2013; Ramos et al., 2018). Studies on entomopathogenic $C$. nymphaeae have focused on its utility and development as a biological control agent of P. praelonga in citrus production (Teixeira et al., 2001; Teixeira et al., 2004). Natural outbreaks of the fungus with high mortality of citrus scale are observed in conventional citrus groves; outbreaks appear to be density dependent with increased prevalence during rainy and warm weather (Mascarin et al., 2016).
In Brazil, C. nymphaeae on citrus scale is colloquially known as the salmão (salmon) fungus-a reference to the characteristic salmon pink color of the conidial masses on infected insects. The salmão fungus, previously $C$. gloeosporioides f. sp. ortheziidae, was transferred to $C$. nymphaeae based on a single locus and without a comparative morphological study (Damm et al., 2012; Marcelino et al., 2008). The designation formae speciales (f. sp.) was dropped by this transfer; therefore, entomopathogenic isolates of $C$. nymphaeae are no longer distinguished by name from its plant pathogenic conspecifics. Because of its potential for biological control, the taxonomy and affinity of the salmão fungus require further study. In this study, through morphological and molecular studies, we take a polyphasic approach to clarifying the taxonomy of this important natural enemy of the citrus scale insect.

\section{Materials and Methods}

\section{Fungal isolation and preservation}

Two isolates of Colletotrichum sp., collected from the citrus scale insect, were purified in vitro on potatodextrose-agar and subsequently deposited in the Laboratório de Patologia e Controle Microbiano, Departamento de Entomologia e Acarologia of the Escola Superior de Agricultura "Luiz de Queiroz", Universidade de São Paulo (USP/ESALQ). Isolate ESALQ 1393 was collected in Limeira, state of Sao Paulo, 8 Feb 2007, Sylvio Baggio s.n., and isolate ESALQ 1368 from Cordeirópolis, state of Sao Paulo, 13 July 2005, Luiz Fernando Padulla s.n. Both isolates were preserved in a lyophilized state and in sterile $10 \%$ glycerol at $-80{ }^{\circ} \mathrm{C}$ as metabolically inactive strains.

\section{Morphological study}

Morphological study and descriptions were made from observations of fungal structures mounted in wa- 
ter on a glass slide. Measurements and light photomicrographs were taken using an Olympus AX70 Provis compound light microscope and Cell $\wedge \mathrm{A}$ analysis image processing software as well as an Olympus SZX16 dissecting microscope. Herbarium acronyms followed those of the Index Herbariorum (Thiers, 2018). Morphological characters were recorded from cultures grown on sabouraud dextrose agar (SDA), oatmeal agar (OA) (Crous et al., 2009), and synthetic nutrient-poor agar medium (SNA) (Nirenberg, 1976) and maintained at $22^{\circ} \mathrm{C}$ with a light regime of $18 \mathrm{~h}$ of darkness and $6 \mathrm{~h}$ of light. Figures were assembled with Adobe Photoshop CS4.

\section{Molecular study}

Genomic DNA was extracted from C. nymphaeae isolates from infected $P$. praelonga individuals collected from citrus orchards in Brazil. One isolate, ARSEF 4360, was obtained from the USDA-ARS Collection of Entomopathogenic Fungal Cultures (ARSEF) and ESALQ 1393 was obtained from the culture collection of the Laboratório de Patologia e Controle Microbiano, Universidade de São Paulo (USP/ESALQ). DNA from ESALQ 1393 and for ARSEF 4360 were extracted as described in Mascarin et al. (2016); genomic DNA was obtained by grinding the mycelium with a plastic pestle inside a $1.5 \mathrm{~mL}$ Eppendorf tube. DNA was then isolated using a commercial plant DNA extraction kit using the standard protocol and eluted with $100 \mu \mathrm{L}$ sterile deionized water.

Three loci were amplified: the entire nrDNA ITS region (ITS1-5.8S-ITS2), actin (ACT) and chitin synthase (CHS-1). The entire nrDNA ITS region (ITS1-5.8S-ITS2) was amplified using primer pairs ITS1F (Gardes and Bruns, 1993) + ITS4 (White et al., 1990), ACT and CHS1 were amplified and sequenced using the primer pairs ACT-512F + ACT-783R and CHS-354R + CHS-79F (Carbone and Kohn, 1999), respectively. Primers and primer sequences are listed in Table 1. PCR was performed under the following conditions for ITS: step 1) $1 \mathrm{~min}$ at $\left.95{ }^{\circ} \mathrm{C}, 2\right) 45 \mathrm{~s}$ at $\left.\left.95^{\circ} \mathrm{C}, 3\right) 40 \mathrm{~s}, 50.8^{\circ} \mathrm{C}, 4\right) 90 \mathrm{~s}$ at $72^{\circ} \mathrm{C}$, 5) return to step 234 times, 6) a final step of $10 \mathrm{~min}$ at $72{ }^{\circ} \mathrm{C}$; for both ACT and CHS-1: step 1) 3 min at $95{ }^{\circ} \mathrm{C}$, 2) $15 \mathrm{~s}$ at $\left.95{ }^{\circ} \mathrm{C}, 3\right) 20 \mathrm{~s}$ at $\left.58{ }^{\circ} \mathrm{C}, 4\right) 1 \mathrm{~min}$ at $\left.72{ }^{\circ} \mathrm{C}, 5\right)$ return to step 234 times, 6) final step of $5 \mathrm{~min}$ at $72^{\circ} \mathrm{C}$. Samples were kept at $10^{\circ} \mathrm{C}$ until electrophoresis was performed on a $1 \%$ agarose TAE gel and visualized under UV light. PCR products were cleaned using a PCR purification kit and sent for sequencing. Sequences were assembled with Sequencher v. 5.3 and aligned manually in MEGA5 (Tamura et al., 2011).

Using the newly obtained sequences as well as sequences from GenBank, including those generated by Damm et al. (2012), five datasets were produced: ITS and TUB2 datasets each having 77 sequences, and glyceraldehyde-3-phosphate dehydrogenase (GADPH), CHS1 and ACT datasets each having 76 sequences. A total of three strains of $C$. nymphaeae isolated from $P$. praelonga were included in the molecular study: ESALQ 1368, ESALQ 1393, ARSEF 4360; however, only the last two, for which multiple loci were obtained, were included in the combined phylogenetic analysis. The taxa and isolates used in the study are shown in Table 2.

The individual ITS, GDPH, CHS-1, ACT, TUB2 matrices were exported in NEXUS format and converted to a combined data file in PAUP* v. 4.0.10b. The individual datasets were analyzed by maximum parsimony using a heuristic search with random addition sequence, TBR swapping and 1,000 heuristic replicates, saving no more than ten best trees per replicate, followed by a final search of saved trees. The phylograms of each dataset were visually inspected for topological congruence and then combined into a single dataset. The combined dataset was bootstrapped $(2,000$ replicates $)$ using the same maximum parsimony search parameters. Following Damm et al. (2012), C. orchidophilum was used as the outgroup for the $C$. acutatum complex. A final phylogram with added bootstrap values was prepared using the Adobe Illustrator Professional. The combined dataset was deposited in TreeBase and can be accessed at http:// purl.org/phylo/treebase/phylows/study/TB2:S18116

\section{Results}

\begin{abstract}
Molecular study
The combined ITS + GDPH + CHS $1+\mathrm{ACT}+\mathrm{TUB} 2$ dataset included 1,661 characters of which 1,233 were constant, 50 were variable but not parsimony-informative, and 378 were parsimony-informative. A total of 600 equally most-parsimonious trees of 432 steps were recovered: a phylogram of one of these trees is shown in Figure 1. Isolates from the citrus scale insect are in a well-supported $C$. nymphaeae clade [97\% bootstrap support (bs)] (Figure 1). The combined dataset recovered two major clades for $C$. nymphaeae with respective bootstrap support values of $86 \%$ and $89 \%$. The citrus
\end{abstract}

Table 1 - Primer sequences and references for loci amplified in this study: ACT = actin, CHS-1= chitin synthase 1 and ITS = Internal transcribed spacer (ITS) situated between the small-subunit ribosomal RNA.

\begin{tabular}{lccc}
\hline Locus & Primers & Sequence (5'- 3') & Reference \\
\hline ACT & ACT-512F & ATG TGC AAG GCC GGT TTC GC & Carbone and Kohn, 1999 \\
\hline CHS-1 & ACT-783R & TAC GAG TCC TTC TGG CCC AT & Carbone and Kohn, 1999 \\
\hline & CHS-354R & TGG AAG AAC CAT CTG TGA GAG TTG & Carbone and Kohn, 1999 \\
\hline ITS & CHS-79F & TGG GGC AAG GAT GCT TGG AAG AAG & Carbone and Kohn, 1999 \\
\hline & ITS1F & CTT GGT CAT TTA GAG GAA GTA A & Gardes and Brunes, 1993 \\
\hline
\end{tabular}


Table 2 - Host information, geographical origin and ITS, ACT, CHS-1, GADPH and TUB2 GenBank accession numbers of the taxa/strains included in the molecular study. Types are designated by an asterisk. Insect hosts are underlined.

\begin{tabular}{|c|c|c|c|c|c|c|c|c|}
\hline Species & Isolate/Strain & Host/substrate & Geographic origin & ITS & ACT & CHS-1 & GADPH & TUB2 \\
\hline C. acerbum & CBS $128530^{*}$ & Malus domestica & New Zealand & JQ948459 & JQ949780 & JQ949120 & JQ948790 & JQ950110 \\
\hline \multirow[t]{6}{*}{ C. acutatum } & CBS 144.29 & Capsicum annuum & Sri Lanka & JQ948401 & JQ949722 & JQ949062 & JQ948732 & JQ950052 \\
\hline & CBS 112996* & Carica papaya & Australia & JQ005776 & JQ005839 & JQ005797 & JQ948677 & JQ005860 \\
\hline & CBS 979.69 & Coffea arabica & Kenya & JQ948400 & JQ949721 & JQ949061 & JQ948731 & JQ950051 \\
\hline & CBS 370.73 & Pinus radiata & New Zealand & JQ948351 & JQ949672 & JQ949012 & JQ948682 & JQ950002 \\
\hline & IMI 336479 & Pistacia vera & Australia & JQ948367 & JQ949688 & JQ949028 & JQ948698 & JQ950018 \\
\hline & CBS 113006 & Protea cynaroides & South Africa & JQ948390 & JQ949711 & JQ949051 & JQ948721 & JQ950041 \\
\hline \multirow[t]{2}{*}{ C. australe } & CBS 116478* & Trachycarpus fortunei & South Africa & JQ948455 & JQ949776 & JQ949116 & JQ948786 & JQ950106 \\
\hline & CBS 131325 & Hakea sp. & Australia & JQ948456 & JQ949777 & JQ949117 & JQ948787 & JQ950107 \\
\hline C. brisbanense & CBS 292.67* & Capsicum annuum & Australia & JQ948291 & JQ949612 & JQ948952 & JQ948621 & JQ949942 \\
\hline C. cosmi & CBS 853.73* & Cosmos sp. & Netherlands & JQ948274 & JQ949595 & JQ948935 & JQ948604 & JQ949925 \\
\hline \multirow[t]{2}{*}{ C. costaricense } & CBS $330.75^{*}$ & Coffea arabica & Costa Rica & JQ948180 & JQ949501 & JQ948841 & JQ948510 & JQ949831 \\
\hline & CBS 211.78 & Coffea sp. & Costa Rica & JQ948181 & JQ949502 & JQ948842 & JQ948511 & JQ949832 \\
\hline C. cuscutae & IMI 304802* & Cuscuta sp. & Dominica & JQ948195 & JQ949516 & JQ948856 & JQ948525 & JQ949846 \\
\hline \multirow[t]{10}{*}{ C. fioriniae } & CBS 125396 & Malus domestica & USA & JQ948299 & JQ949620 & JQ948960 & JQ948629 & JQ949950 \\
\hline & IMI 345578 & Fragaria $\times$ ananassa & New Zealand & JQ948330 & JQ949655 & JQ948995 & JQ948660 & JQ949981 \\
\hline & CBS 126523 & Berberis sp. & Netherlands & JQ948322 & JQ949643 & JQ948983 & JQ948652 & JQ949973 \\
\hline & CBS 786.86 & Malus sylvestris & Italy & JQ948303 & JQ949624 & JQ948964 & JQ948633 & JQ949954 \\
\hline & CBS 112995 & Malus domestica & USA & JQ948298 & JQ949619 & JQ949289 & JQ948628 & JQ949949 \\
\hline & CBS 981.69 & Coffea arabica & Angola & JQ948327 & JQ949648 & JQ948988 & JQ948657 & JQ949978 \\
\hline & CBS 200.35 & Rubus sp. & USA & JQ948293 & JQ949614 & JQ948954 & JQ948623 & JQ949944 \\
\hline & CBS 490.92 & Solanum lycopersicum & New Zealand & JQ948326 & JQ949647 & JQ948987 & JQ948656 & JQ949977 \\
\hline & CBS 129948 & Tulipa sp. & UK & JQ948344 & JQ949665 & JQ949005 & JQ948674 & JQ949995 \\
\hline & CBS 119292 & Vaccinium sp. & New Zealand & JQ948313 & JQ949634 & JQ948974 & JQ948643 & JQ949964 \\
\hline \multirow[t]{4}{*}{ C. godetiae } & CBS $133.44^{*}$ & Clarkia hybrida & Denmark & JQ948402 & JQ949723 & JQ949063 & JQ948733 & JQ950053 \\
\hline & CBS 129934 & Prunus dulcis & Israel & JQ948431 & JQ949752 & JQ949092 & JQ948762 & JQ950082 \\
\hline & CBS 862.70 & Sambucus nigra & etherlands & JQ948437 & JQ949758 & JQ949098 & JQ948768 & JQ950088 \\
\hline & CBS 127561 & Ugni molinae & Chile & JQ948442 & JQ949763 & JQ949103 & JQ948773 & JQ950093 \\
\hline C. guajavae & IMI 350839* & Psidium guajava & India & JQ948270 & JQ949591 & JQ948931 & JQ948600 & JQ949921 \\
\hline C. indonesiense & CBS $127551^{*}$ & Eucalyptus sp. & Indonesia & JQ948288 & JQ949609 & JQ948949 & JQ948618 & JQ949939 \\
\hline \multirow[t]{2}{*}{ C. johnstonii } & IMI 357027 & Citrus sp. & New Zealand & JQ948443 & JQ949764 & JQ949104 & JQ948774 & JQ950094 \\
\hline & CBS $128532^{*}$ & Solanum lycopersicum & New Zealand & JQ948444 & JQ949765 & JQ949105 & JQ948775 & JQ950095 \\
\hline C. kinghornii & CBS $198.35^{*}$ & Phormium sp. & UK & JQ948454 & JQ949775 & JQ949115 & JQ948785 & JQ950105 \\
\hline \multirow[t]{2}{*}{ C. laticiphilum } & CBS 112989* & Hevea brasiliensis & India & JQ948289 & JQ949610 & JQ948950 & JQ948619 & JQ949940 \\
\hline & CBS 129827 & Hevea brasiliensis & Colombia & JQ948290 & JQ949611 & JQ948951 & JQ948620 & JQ949941 \\
\hline C. limetticola & CBS $114.14^{*}$ & Citrus aurantifolia & USA, Florida & JQ948193 & JQ949514 & JQ948854 & JQ948523 & JQ949844 \\
\hline \multirow[t]{3}{*}{ C. lupini } & CBS 129944 & Cinnamomum verum & Portugal & JQ948178 & JQ949499 & JQ948839 & JQ948508 & JQ949829 \\
\hline & CBS 513.97 & Lupinus polyphyllus & Costa Rica & JQ948157 & JQ949478 & JQ948818 & JQ948487 & JQ949808 \\
\hline & CBS 466.76 & Manihot utilissima & Rwanda & JQ948160 & JQ949481 & JQ948821 & JQ948490 & JQ949811 \\
\hline C. melonis & CBS $159.84^{*}$ & Cucumis melo & Brazil & JQ948194 & JQ949515 & JQ948855 & JQ948524 & JQ949845 \\
\hline \multirow[t]{10}{*}{ C. nymphaeae } & CBS 130.80 & Anemone sp. & Italy & JQ948226 & JQ949547 & JQ948887 & JQ948556 & JQ949877 \\
\hline & IMI 379162 & Capsicum annuum & Zimbabwe & JQ948218 & JQ949539 & JQ948879 & JQ948548 & JQ949869 \\
\hline & CBS 129945 & Olea europaea & Portugal & JQ948201 & JQ949548 & JQ948888 & JQ948531 & JQ949852 \\
\hline & IMI 360386 & Pelargonium graveolens & India & JQ948206 & JQ949527 & JQ948867 & JQ948536 & JQ949857 \\
\hline & ESALQ 1368 & Praelongorthezia praelonga & Brazil & - & - & - & - & KJ509200 \\
\hline & ESALQ 1393* & P. praelonga & Brazil & MG547976 & MG572798 & MG572799 & - & KJ509199 \\
\hline & ARSEF 4360 & $\underline{P . \text { praelonga }}$ & Brazil & EF593371 & MG572800 & MG572801 & EF593346 & EF593327 \\
\hline & CBS 361.79 & Anemone coronaria & Netherlands & JQ948248 & JQ949569 & JQ948909 & JQ948578 & JQ949899 \\
\hline & CBS 126377 & Fragaria $\times$ ananassa & Netherlands & JQ948233 & JQ949554 & JQ948894 & JQ948563 & JQ949884 \\
\hline & CBS 112202 & Fragaria sp. & Spain & JQ948234 & JQ949555 & JQ948895 & JQ948564 & JQ949885 \\
\hline \multirow[t]{3}{*}{ C. nymphaeae } & CBS 129926 & Litter & Thailand & JQ948216 & JQ949537 & JQ948877 & JQ948546 & JQ949867 \\
\hline & CBS 516.78 & Nuphar luteum & Netherlands & JQ948198 & JQ949519 & JQ948859 & JQ948528 & JQ949849 \\
\hline & CBS $515.78^{*}$ & Nymphaea alba & Netherlands & JQ948197 & JQ949518 & JQ948858 & JQ948527 & JQ949848 \\
\hline
\end{tabular}


Table 2 - Continuation.

\begin{tabular}{|c|c|c|c|c|c|c|c|c|}
\hline & CSL 455 & Photinia sp. & UK & JQ948217 & JQ949538 & JQ948878 & JQ948547 & JQ949868 \\
\hline & EMA26 & Praelongorthezia praelonga & Brazil & EF593372 & - & - & EF593347 & EF593328 \\
\hline & CBS 112992 & Protea magnifica & South Africa & JQ948207 & JQ949528 & JQ948868 & JQ948537 & JQ949858 \\
\hline \multirow[t]{2}{*}{ C. orchidophilum } & CBS 631.80 & Ascocenda sp. & USA & JQ948152 & JQ949473 & JQ948813 & JQ948482 & JQ949803 \\
\hline & CBS $632.80^{*}$ & Dendrobium sp. & USA & JQ948151 & JQ949472 & JQ948812 & JQ948481 & JQ949802 \\
\hline \multirow[t]{2}{*}{ C. paxtonii } & CBS 502.97 & Musa nana & West Indies & JQ948286 & JQ949607 & JQ948947 & JQ948616 & JQ949937 \\
\hline & IMI 165753* & Musa sp. & Saint Lucia & JQ948285 & JQ949606 & JQ948946 & JQ948615 & JQ949936 \\
\hline \multirow[t]{3}{*}{ C. phormii } & CBS 118191 & Phormium sp. & South Africa & JQ948453 & JQ949774 & JQ949114 & JQ948784 & JQ950104 \\
\hline & CBS 118197 & Phormium sp. & New Zealand & JQ948450 & JQ949771 & JQ949111 & JQ948781 & JQ950101 \\
\hline & CBS 483.82 & Phormium tenax & New Zealand & JQ948451 & JQ949772 & JQ949112 & JQ948782 & JQ950102 \\
\hline C. pyricola & CBS $128531^{*}$ & Pyrus communis & New Zealand & JQ948445 & JQ949766 & JQ949106 & JQ948776 & JQ950096 \\
\hline \multirow[t]{2}{*}{ C. rhombiforme } & CBS 129953 * & Olea europaea & Portugal & JQ948457 & JQ949778 & JQ949118 & JQ948788 & JQ950108 \\
\hline & CBS 131322 & Vaccinium macrocarpum & USA & JQ948458 & JQ949779 & JQ949119 & JQ948789 & JQ950109 \\
\hline \multirow[t]{2}{*}{ C. salicis } & CBS 465.83 & Araucaria excelsa & USA & JQ948468 & JQ949789 & JQ949129 & JQ948799 & JQ950119 \\
\hline & CBS $607.94^{*}$ & Salix sp. & Netherlands & JQ948460 & JQ949781 & JQ949121 & JQ948791 & JQ950111 \\
\hline \multirow[t]{2}{*}{ C. scovillei } & CBS $126529^{*}$ & Capsicum sp. & Indonesia & JQ948267 & JQ949588 & JQ948928 & JQ948597 & JQ949918 \\
\hline & CBS 120708 & Capsicum annuum & Thailand & JQ948269 & JQ949590 & JQ948930 & JQ948599 & JQ949920 \\
\hline \multirow[t]{2}{*}{ C. simmondsii } & CBS 294.67 & Carica papaya & Australia & JQ948277 & JQ949598 & JQ948938 & JQ948607 & JQ949928 \\
\hline & CBS 126524 & Cyclamen sp. & Netherlands & JQ948281 & JQ949602 & JQ948942 & JQ948611 & JQ949932 \\
\hline C. sloanei & IMI 364297* & Protea cynaroides & Malaysia & JQ948287 & JQ949608 & JQ948948 & JQ948617 & JQ949938 \\
\hline \multirow[t]{2}{*}{ C. tamarilloi } & CBS $129814^{*}$ & Theobroma cacao & Colombia & JQ948184 & JQ949505 & JQ948845 & JQ948514 & JQ949835 \\
\hline & CBS 129956 & Solanum betaceum & Colombia & JQ948190 & JQ949511 & JQ948851 & JQ948520 & JQ949841 \\
\hline C. walleri & CBS $125472^{*}$ & Coffea sp. & Vietnam & JQ948275 & JQ949596 & JQ948936 & JQ948605 & JQ949926 \\
\hline C. sp. & CBS 129810 & Solanum betaceum & Colombia & JQ948179 & JQ949500 & JQ948840 & JQ948509 & JQ949830 \\
\hline
\end{tabular}

scale insect isolates are in the same clade as isolate CBS 515.78 , the type of $C$. nymphaeae designated as $C$. nymphaeae var. nymphaeae in Figure 1 . In the beta-tubulin dataset, a single nucleotide mutation was present in all four entomopathogenic isolates of $C$. nymphaeae: this mutation is shared only by one other species, $C$. australe, which is also in the $C$. acutatum complex, but distantly related to $C$. nymphaeae (Table 3; Figure 1). Isolates of $C$. nymphaeae from the citrus scale insect differed from all other $C$. nymphaeae isolates by two nucleotide changes in the CHS-1 gene (Table 4). Insect pathogenic C. nymphaeae, colloquially referred to as the salmão fungus is described herein as a new variety of $C$. nymphaeae.

\section{Taxonomy}

Collectrichum nymphaeae var. entomophilum A.A. Wynns \& I. Delalibera, var. nov.

MycoBank $823559 \quad$ Figure 2A-H

$=C$. gloeosporioides f. sp. ortheziidae (Marcelino et al., 2008)

Etymology: The varietal epithet, which means insect loving, indicates its predilection for insects.

Type: Limeira, state of São Paulo, isolated from a Praelongorthezia praelonga (Hemiptera: Ortheziidae) cadaver, 8 Feb 2007, Silvio Baggio s.n. Holotype, ESA 142963 permanently preserved in a metabolically inactive state as a dried culture in microscope slides of ESALQ 1393-H deposited in the herbarium of Escola
Table 3 - Beta-tubulin gene sequence segment showing single nucleotide mutation present in all four isolates of $C$. nymphaeae var. entomophilum and shared by only one other taxon (C. australe) within the $C$. acutatum complex.

\begin{tabular}{lcc}
\hline Taxon & Isolate & Beta-tubulin sequence \\
\hline \multicolumn{1}{l}{ C. nymphaeae } & & \\
\hline \multicolumn{1}{c}{ var. entomophilum } & ARSEF 4360 & TCAC-TCGTTCTCCAGTG \\
& EMA 26 & TCAC-TCGTTCTCCAGTG \\
& ESALQ 1368 & TCAC-TCGTTCTCCAGTG \\
ESALQ 1393 & TCAC-TCGTTCTCCAGTG \\
var. nymphaeae & IMI 360386 & TCAC-TCGTCCTCCAGTG \\
\hline C. australe & & \\
\hline & CBS 515.78 & TCAC-TCGTCCTCCAGTG \\
\hline & CBS 131325 116478 & TCTC-TTGTTCTCCAGTG \\
\hline
\end{tabular}

Table 4 - CHS-1 gene nucleotide differences between C. nymphaeae var. nymphaeae and $C$. numphaeae var. entomophilum.

\begin{tabular}{lccc}
\hline Taxon & Isolate & \multicolumn{2}{c}{$\begin{array}{c}\text { Mutations and their position in the CHS- } \\
\text { 1 gene sequence }\end{array}$} \\
\hline C. nymphaeae & & 217 & 247 \\
\hline var. entomophilum & ARSEF 4360 & C & T \\
& ESALQ 1393 & C & T \\
\hline var. nymphaeae & CBS 515.78 & T & C \\
& IMI 360386 & T & C \\
& CBS 130.80 & T & C \\
\hline
\end{tabular}




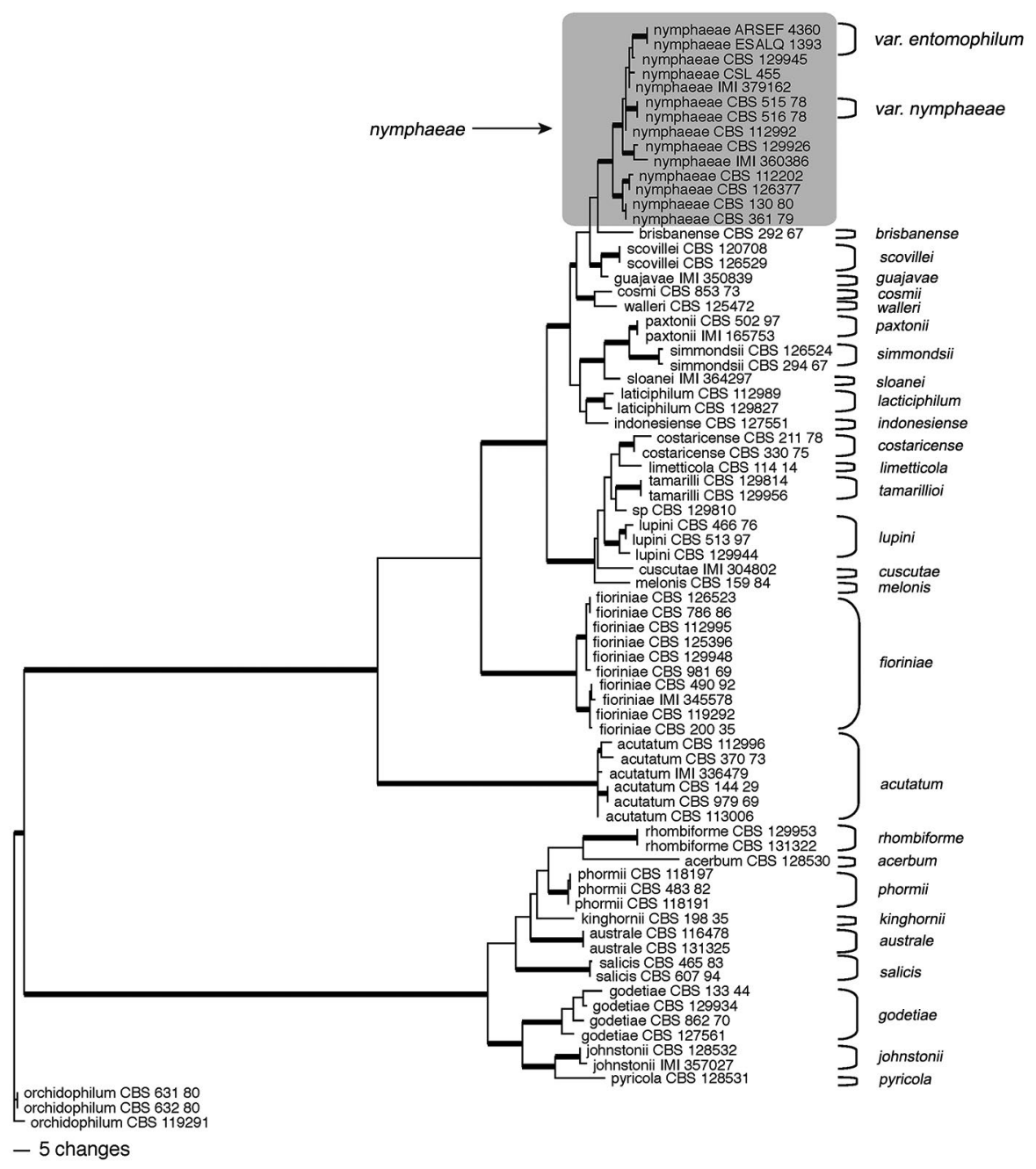

Figure 1 - One of 9,139 equally most-parsimonious phylograms from a maximum parsimony analysis of ITS + GADPH + CHS- $1+$ ACT + TUB2 combined sequence data from 73 isolates within the Colletotrichum acutatum species complex. Thickened branches lead to nodes with bootstrap support values $\geq 70 \%$ (2000 replicates).

Superior de Agricultura "Luiz de Queiroz" from the Universidade de São Paulo (USP/ESALQ/ESA - http://splink. cria.org.br/manager/detail?setlang $=$ pt\&resource $=\mathrm{ESA})$. Ex-holotype cultures are stored in lyophilized inactive state as ESALQ $1393-H$ in the Collection of Entomopathogenic Fungi, Laboratório de Patologia e Controle Microbiano, Escola Superior de Agricultura "Luiz de Queiroz", Universidade de São Paulo (USP/ESALQ), Piracicaba, state of São Paulo, and as CG1414 in the Invertebrate-Associated Fungal Collection of Embrapa (CG), Embrapa Genetic Resources and Biotechnology, DF-70770-970, Brazil (http://www.wfcc.info/ccinfo/collection/by_id/712).

Colonies on OA $10.5 \mathrm{~cm}$ at seven days, a low cottony mycelium with concentric alternating white, salmon and grey rings (Figure 2A); salmon color from numerous small acervuli oozing conidia; culture in reverse pale greyish-brown with a greyish-salmon center. On
SNA $9 \mathrm{~cm}$ at seven days, mycelium uniform, low, white, opaque (Figure 2B); colorless in reverse; acervuli few, colorless. Vegetative hyphae 1.1-8.0 $\mu \mathrm{m}$, average $3.2 \mu \mathrm{m}$. Conidiomata acervular. Conidiogenous cells hyaline, 4.3 $18.2 \times 2.2-4.1 \mu \mathrm{m}$, ave. $11.3 \times 3.2 \mu \mathrm{m}$. Conidia hyaline, pale orange in mass, 1-celled, $6.4-16.0 \times 3.2-5.4 \mu \mathrm{m}$, ave. $10.9 \times 4.2 \mu \mathrm{m}(\mathrm{n}=41), \mathrm{L} / \mathrm{W}$ ratio 2.6 , cylindrical to ellipsoidal with ends broadly or acutely rounded (Figure 2C). Appressoria reniform, brown, smooth-walled, margin entire, 5.9 - $9.8 \times 3.9-7.7 \mu \mathrm{m}$ (Figure $2 \mathrm{~F}$ ). Setae on OA rare, brown, thick-walled, irregular margin, tip acute, 68.5 - $73.1 \times 3.4-3.7 \mu \mathrm{m}$ (Figure 2D and E).

Host: Praelongorthezia praelonga. Figure 2G-H.

Diagnosis: Collectrichum nymphaeae var. entomophilum is distinguished morphologically from $C$. nymphaeae var. nymphaeae by its smaller conidia $6.4-16 \times 3.2-5.4$ $\mu \mathrm{m}$, ave. $10.9 \times 4.2 \mu \mathrm{m}$ and the presence of setae (Table 5). The average length and width of conidia reported for 
Table 5 - Colony characteristics on oatmeal agar (OA) and synthetic nutrient-poor agar medium (SNA) at seven days and morphological measurements of SNA cultures for $C$. nymphaeae var. entomophilum and C. nymphaeae var. nymphaeae. Information not provided is indicated by a dash (-).

\begin{tabular}{lccccc}
\hline \multirow{2}{*}{ Colletotrichum nymphaeae } & \multicolumn{3}{c}{ Colony diameter $(\mathrm{cm}) 7$ days } & \multicolumn{2}{c}{ Conidia } \\
\cline { 2 - 5 } & SNA & OA & Length [ave] $(\mu \mathrm{m})$ & Width [ave] $(\mu \mathrm{m})$ & petae \\
\hline var. entomophilum & 9.0 & 10.5 & $6.4-14.9[10.9]$ & $3.5-4.9[4.2]$ & present \\
\hline var. nymphaeae (CBS 515.78) & 3.6 & 5.0 & $12.3-32.7[17.2]$ & $3.7-6.9[5.0]$ & absent \\
\hline nymphaeae (Damm et al., 2012) & $1.65-2.6$ & $1.45-2.9$ & $10-19.5[16.1]$ & $3-6[4.9]$ & absent \\
\hline nymphaeae (Hemmi and Kawasi, 1954) & - & - & $9-17[-]$ & $3-6[-]$ & present \\
\hline
\end{tabular}

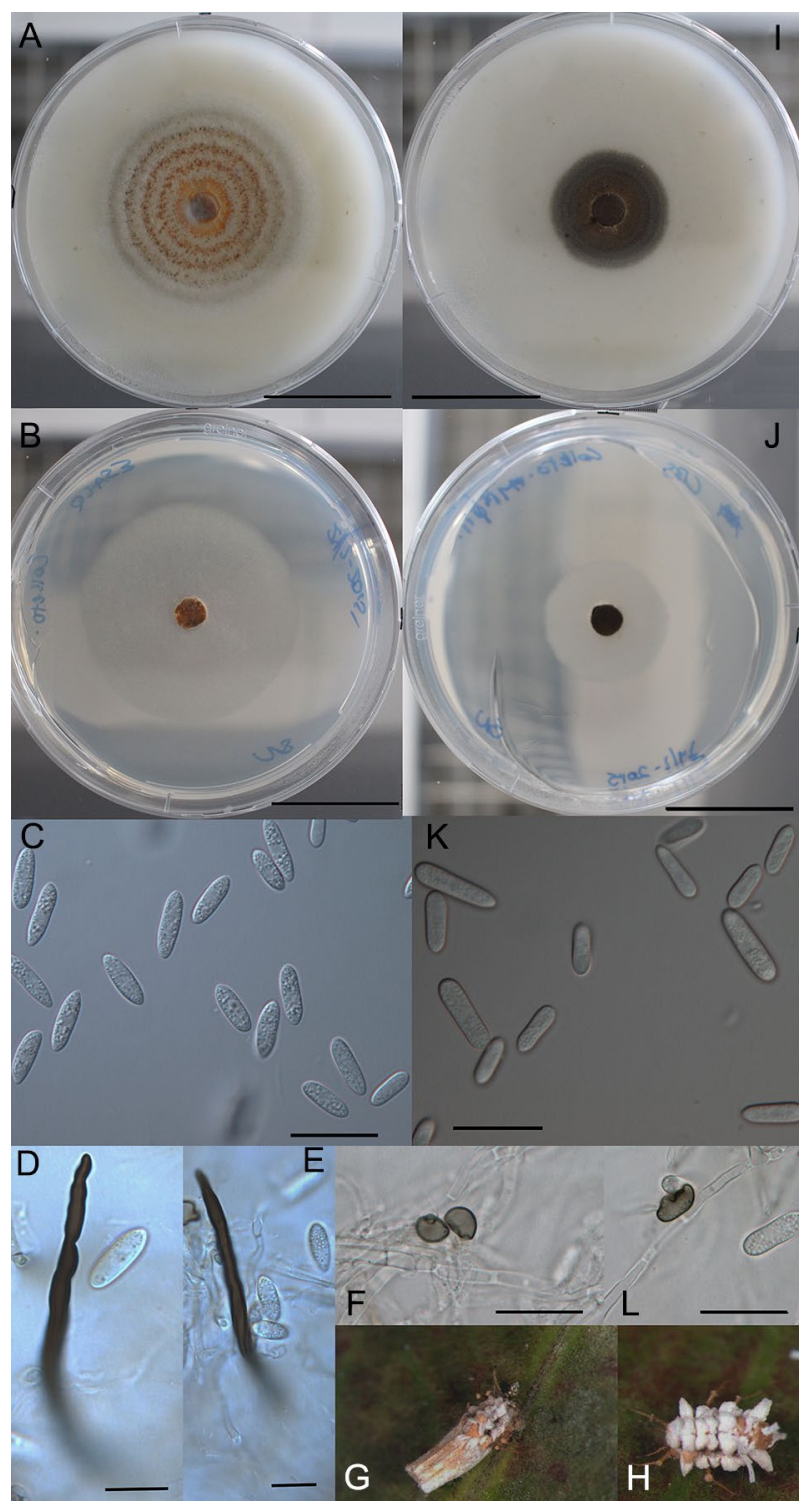

Figure 2 - A - H) Colletotrichum nymphaeae var. entomophilum (ESALQ 1393). A) culture on OA after eight days. B) culture on SNA after seven days. C) conidia. D - E) setae. F) appressorium. G - H) infected, sporulating, Praelongortheziidae praelonga cadavers. I - L) Plant pathogenic $C$. nymphaeae var. nymphaeae (CBS 515.78). I) culture on OA after seven days. J) culture on SNA after seven days. K) conidia. L) appressoria. Scale bars: A-J $=20 \mu \mathrm{m} ; \mathrm{C}-\mathrm{F}, \mathrm{K}-\mathrm{L}=10 \mu \mathrm{m}$.
C. nymphaeae is $16.1 \times 4.9 \mu \mathrm{m}$ with the exception of one strain, CBS 526.77, which was reported as having conidia measuring 9 - $13 \times 3-4.5 \mu \mathrm{m}$ (Damm et al., 2012). In addition to morphological differences, $C$. nymphaeae var. entomophilum is distinct from all other isolates of $C$. nymphaeae by a single nucleotide mutation in the betatubulin gene (Table 3), two nucleotide mutations in the CHS-1 gene (Table 4) and by its insect, rather than plant host preference.

Additional notes. Colletotrichum nymphaeae var. entomophilum more closely matches the description of Gloeosporium (Colletotrichum) nymphaeae Hemmi and Kawase, isolated from leaves of the Nymphaea in Japan (Hemmi and Kawase, 1954), than the description of $C$. nymphaeae sensu Damm et al. (2012). Hemmi and Kawase report dark brown thick-walled setae, $19-76 \mu \mathrm{m}$ long and conidia ranging from $9-17 \times 3-6 \mu \mathrm{m}$. Unfortunately, the specimens cited by Hemmi and Kawasi (1954) cannot be found and no living cultures exist. According to Damm et al. (2012), C. nymphaeae as currently circumscribed is probably not the same organism described by Hemmi and Kawasi primarily because setae have never been observed in $C$. nymphaeae, neither by Damm et al. (2012) nor by van der Aa (1978); however, Batista and Bezerra (1966) described abundant setae in citrus orthezia insects infected by a "special strain" of $C$. gloeosporioides. Batista and Bezerra (1966) did not cite specimens in their study; however, the special strain they discovered and reported for the first time was likely C. nymphaeae var. entomophilum.

Additional specimens were examined at Cordeirópolis, São Paulo, 13 July 2005, L.F. Padulla s.n., ESALQ 1368, and Rio de Janeiro, state of Rio de Janeiro, Feb 1994, C.F. Robbs ARSEF 4360.

\section{Colletotrichum nymphaeae var. nymphaeae}

(Pass.) Aa, Netherlands Journal of Plant Pathology, Supplement 184 (3): 110, Fig. 20. 1978; 三Ascochyta nymphaeae Pass., Hedwigia 16:120, 1877. Lectotype, Italy, Parma, from the leaf of Nymphaea alba (Nymphaeaceae), summer 1875, G. Passerini, 176820 (K, non vidi); Epitype, Netherlands, Ubbergen, Oude Waal near Nijmegen, from the leaf spots of Nymphaeae alba, 7 Aug 1978, G. van der Velde (CBS H-20787), ex-epitype culture CBS 515.78 ! = van der Aa No. 657.

Figure 2I-L

Colonies on OA $3.6 \mathrm{~cm}$ in seven days, a low felty mycelium with white, floccose center (Figure 2I-J); acer- 
vuli salmon colored; culture in reverse dark brownishgrey. On SNA $5.0 \mathrm{~mm}$ in seven days, flat with a diffuse but well-defined irregular margin, hyaline to whitish; acervuli scattered, colorless. Vegetative hyphae $1.1-3.2$ $\mu \mathrm{m}$, septate, branched. Conidiogenous cells hyaline, 13.3 $20.9 \times 3.2-3.8 \mu \mathrm{m}$, ave. $17.1 \times 3.6 \mu \mathrm{m}$. Conidia hyaline, pale orange in mass, 1-celled, $12.3-24(-32.7) \times 3.7-6.9$ $\mu \mathrm{m}$, ave. $17.2 \times 5.0 \mu \mathrm{m}(\mathrm{n}=31), \mathrm{L} / \mathrm{W}$ ratio 3.4 , cylindrical, frequently narrowly obovate (Figure $2 \mathrm{~K}$ ). Appressoria reniform to elongate with crenulate margins, brown, $7.6-10.5 \times 4.8-9.8 \mu \mathrm{m}$ (Figure 2L). Setae not observed.

\section{Discussion}

Delineating taxa and resolving the relationships within Colletotrichum is a challenge because of high sequence homogeneity and morphological uniformity within the genus. For this reason, multiple genes are required not just for elucidating the relationships between taxa but also for species identification. Using DNA sequence data from five gene regions: GDPH + TUB2 + ITS + ACT + CHS-1, we found strong support for including the insect pathogenic isolates of $C$. nymphaeae (formerly $C$. gloeosporioides f. sp. ortheziidae in the species $C$. nymphaeae $(97 \%$ bs) (Figure 1). On the basis of morphological and molecular characters we recognize the insect pathogenic isolates of $C$. nymphaeae as a new variety and designate the name $C$. nymphaeae var. entomophilum. A single nucleotide mutation in the GADPH gene, two mutations in CHS-1, and the presence of setae readily distinguishes this new variety from its closest relatives. Once additional informative markers are found for Colletotrichum, it may be possible to better resolve the relationships within $C$. nymphaeae and determine if $C$. nymphaeae var. entomophilum should continue to be recognized as a variety or as a distinct species. Until then, the circumscription of $C$. nymphaeae sensu Damm et al., 2012, should be modified to include setae. Setae are present in C. nymphaeae var. entomophilum but have so far not been observed in plant symbiont isolates of $C$. nymphaeae. In order to clarify their taxonomic utility, further studies should be conducted to confirm the presence of setae in situ and to elucidate any effects that sub-culturing or other environmental conditions might have on the production of setae in vitro.

Both entomopathogenic Colletotrichum taxa, $C$. nymphaeae var. entomophilum and $C$. fiorniae, display remarkably diverse lifestyle strategies with the ability to live as insect pathogens, plant pathogens and endophytes. At least 50 phytopathogenic isolates of $C$. fiorinae have been identified by DNA sequencing (Damm et al., 2012). Additionally, C. fiorinae has been isolated from fruit rot and detected as an endophyte in 28 species of plants in the region of epizootic outbreaks on the hemlock scale insect (Damm et al., 2012; Marcelino et al., 2009). Colletotrichum nymphaeae var. entomophilum has also been recovered as an endophyte but only in plants inoculated with the fungus (Marcelino et al., 2009). The occurrence of $C$. nymphaeae var. entomophilum in nature, either as an endophyte or on additional insect hosts has not been studied. This is an area of research that merits prompt attention given that endophytic entomopathogens may serve as defensive mutualists when living in plant tissue (Bultman and Faeth, 2002; Crouch et al., 2014; Redman et al., 2002). For example, if C. nymphaeae var. entomophilum occurs endophytically in nature, it may potentially play a larger role in controlling citrus scale and other sap-sucking insects than previously realized.

The significance of the varied lifestyle strategies of C. fiorniae and $C$. nymphaeae var. entomophilum has previously been downplayed because the insect hosts of these fungi are plant sucking insects (Damm et al., 2012). A close relationship between insect pathogenic fungi and grass endosymbionts has previously been shown (Spatafora et al., 2007). Comparative genomic analyses showed that insect pathogenic Metarhizium spp. are more closely related to endophytes and plant pathogens compared to animal pathogens (Gao et al., 2011). The evolutionary transition from plant pathogen to endophyte in Colletotrichum is thought to occur relatively easily; for example, in at least one species of Colletotrichum, a single-locus mutation converts the fungus from a symptomatic plant pathogen to an endophyte. However, transitioning from plant pathogen or endophyte to a pathogen of plant sucking insects may represent a more significant and complex physiological shift than is assumed with genes co-opted, evolved or acquired by horizontal gene transfer from a plant-associated fungus (Barelli et al., 2016). Gene expression in Colletotrichum is highly dependent on plant signaling (Crouch et al., 2014; O'Connell et al., 2012). In vitro signaling has been shown to be markedly different from in planta signaling even from the point of spore germination to the ultimate necrotrophic phase characteristic of plant pathogens (O'Connell et al., 2012). Transcriptomic studies comparing gene expression of the entomopathogenic Colletotrichum taxa in planta, in insecta and in vitro may provide insight into the mechanisms behind inter-kingdom host shifts or the potential of a dual life style as insect pathogens and endophytes; in turn, these insights may help to determine how much or little weight should be given to host preferences for delimiting Colletotrichum taxa.

Much remains to be known about $C$. nymphaeae var. entomophilum. For example, how it is dispersed, its insect host-range, if it occurs endophytically both in nature and in citrus orchards and if endophytic growth affects the fitness of the citrus scale insect. Improved development of this important natural enemy should include studies on the below ground control of Ortheziidae scale insects since these insects also live in soil litter in humid habitats and feed on fungi, mosses and plant roots (Kondo et al., 2013). Entomopathogenic endophytes are well-known among the fungi, especially from the order Hypocreales e.g., Acremonium, Beauveria, Clonostachys, Isaria, Lecanicillium, Verticillium (Vega, 
2008; Vega et al., 2008); however, few are aware that the common plant pathogenic genus Colletotrichum (Glomerellales) also contains endophytic entomopathogens. Clarifying the taxonomy of $C$. nymphaeae var. entomophilum may provide the impetus for further research on this overlooked entomopathogenic fungus. The assignment of a formal name along with morphological and molecular characterization provides a solid framework for facilitating the evaluation and approval of this important fungus for biological control.

\section{Acknowledgments}

This study is a part of the IMBICONT research project (Improved Biological Control for IPM in Fruits and Berries) (project number 1024151001) funded by the Innovation fund, Denmark.

\section{Authors' Contributions}

Conceptualization: Wynns, A.A.; Delalibera, I. Data acquisition: Wynns, A.A.; Delalibera, I.; Jensen, A.A. Data analysis: Wynns, A.A. Design of methodology: Wynns, A.A.; Delalibera, I. Writing and editing: Wynns, A.A.; Delalibera, I.; Jensen, A.A.; Eilenberg, E.

\section{References}

Barelli, L.; Moonjely, S.; Behie, S.W.; Bidochka, M.J. 2016. Fungi with multifunctional lifestyles: endophytic insect pathogenic fungi. Plant Molecular Biology 90: 657-664.

Batista, A.C.; Bezerra J.L. 1966. On the parasitism of Colletotrichum gloeosporioides and other fungi in Orthezia praelonga Douglas. Brotéria 35: 68-74 (in Portuguese, with abstract in English)

Bultman, T.L.; Faeth, S.H. 2002. Endophytic fungi and interactions among host plants, herbivores, and natural enemies. p. 89123. In: Tscharntke, T.; Hawkins, B.A., eds. Multitrophic level interactions. Cambridge University Press, Cambridge, England.

Carbone, I.; Kohn, L.M. 1999. A method for designing primer sets for speciation studies in filamentous ascomycetes. Mycologia 91: 553-556. DOI: $10.2307 / 3761358$

Crouch, J.; O'Connell, R.; Gan, P.; Buiate, E.; Torres, M.F.; Beirn, L.; Shirasu, K.; Vaillancourt, L. 2014. The genomics of Colletotrichum. p. 69-102. In: Dean, R.A.; Lichens-Park, A.; Kole, C., eds. Genomics of plant-associated fungi: monocot pathogens. Springer, Heidelberg, Germany. DOI: 10.1007/9783-662-44053-7_3

Crous, P.W.; Verkley, G.J.M.; Groenewald, J.Z.; Samson, R.A. 2009. Centraalbureau voor Schimmelcultures, Utrecht, Netherlands. (Fungal Biodiversity 269. CBS Laboratory Manual Series 1).

Damm, U.; Cannon, P.F.; Woudenberg, J.H.C.; Crous, P.W. 2012. The Colletotrichum acutatum species complex. Studies in Mycology 73: 37-113. DOI: 10.3114/sim0010

Gao, Q.; Jin, K.; Ying, S.H.; Zhang, Y.; Xiao, G.; Shang, Y.; Duan, Z.; Hu, X.; Xie, X.Q.; Zhou, G.; Peng, G. 2011. Genome sequencing and comparative transcriptomics of the model entomopathogenic fungi Metarhizium anisopliae and M. acridum. Plos Genetics 7: e1001264. DOI: 10.1371/journal.pgen.1001264
Garcia-Roa, F.A. 1995. Management of Orthezia praelonga, A Citrus Pest $=$ Manejo de Orthezia praelonga, una Plaga de los Cítricos. CO-BAC, Santafé de Bogotá, Bogotá, Colombia (in Spanish).

Gardes, M.; Bruns, T.D. 1993. ITS primers with enhanced specificity for basidiomycetes: application to the identification of mycorrhizae and rusts. Molecular Ecology 2: 113-118. DOI: 10.1111/j.1365-294X.1993.tb00005.x

Hanlin, R.T. 1998. Combined Keys to Illustrated Genera of Ascomycetes. APS Press, St. Paul, MN, USA.

Hemmi, T.; Kawase, Y. 1954. On a new anthracnose of water lily caused by Gloeosporium nymphaeae sp. Bulletin of Naniwa University 4: 1-6.

Kondo, T.; Peronti, A.L.; Kozár, F.; Szita, É. 2013. The citrus orthezia, Praelongorthezia praelonga (Douglas) (Hemiptera: Ortheziidae), a potential invasive species. p. 301-319. In: CABI, Wallingford, UK. (CABI Invasive Species Series, 3).

MacKenzie, S.J.; Peres, N.A.; Barquero, M.P.; Arauz, L.F.; Timmer, L.W. 2009. Host range and genetic relatedness of Colletotrichum acutatum isolates from fruit crops and leatherleaf fern in Florida. Phytopathology 99: 620-631. DOI: 10.1094/ phyto-99-5-0620

Manamgoda, D.; Udayanga, D.; Cai, L.; Chukeatirote, E.; Hyde, K. 2013. Endophytic Colletotrichum from tropical grasses with a new species C. endophytica. Fungal Diversity 61:107-115. DOI: 10.1007/s13225-013-0256-3

Marcelino, J.; Giordano, R.; Gouli, S.; Gouli, V.; Parker, B.L.; Skinner, M.; TeBeest, D.; Cesnik, R. 2008. Colletotrichum acutatum var. fioriniae (teleomorph: Glomerella acutata var. fioriniae var. nov.) infection of a scale insect. Mycologia 100: 353-374

Marcelino, J.A.; Gouli, S.; Parker, B.L.; Skinner, M.; Schwarzberg, L.; Giordano, R. 2009. Host plant associations of an entomopathogenic variety of the fungus, Colletotrichum acutatum, recovered from the elongate hemlock scale, Fiorinia externa. Journal of Insect Science 9: 25. DOI: 10.1673/031.009.2501

Mascarin, G.M.; Guarín-Molina, J.H.; Arthurs, S.P.; Humber, R.A.; Andrade, R.M.; Demétrio, C.G.B.; Delalibera, I. 2016. Seasonal prevalence of the insect pathogenic fungus Colletotrichum nymphaeae in Brazilian citrus groves under different chemical pesticide regimes. Fungal Ecology 22: 4345.

Nirenberg, H. 1976. Studies on morphological and biological differentiation in the Liseola section of the genus Fusarium = Untersuchungen Über die morphologische und biologische differenzierung in der Fusarium-section Liseola. Mitteilungen, Biologische Bundesanstalt fur Land und Forstwirtschaft 169: 1-117 (in German).

O'Connell, R.J.; Thon, M.R.; Hacquard, S.; Amyotte, S.G.; Kleemann, J.; Torres, M.F.; Damm, U.; Buiate, E.A.; Epstein, L.; Alkan, N.; Altmüller, J. 2012. Lifestyle transitions in plant pathogenic Colletotrichum fungi deciphered by genome and transcriptome analyses. Nature Genetics 44: 1060-1065.

Photita, W.; Taylor, P.W.J.; Ford, R.; Hyde, K.D.; Lumyong, S. 2005. Morphological and molecular characterization of Colletotrichum species from herbaceous plants in Thailand. Fungal Diversity 18: 117-133. 
Ramos, A.S.; Lemos, R.N.; Costa, V.A.; Peronti, A.L.; Silva, E.A.; Mondego, J.M.; Moreira, A.A. 2018. Hymenopteran parasitoids associated with scale insects (Hemiptera: Coccoidea) in tropical fruit trees in the eastern Amazon, Brazil. Florida Entomologist 101: 273-278.

Redman, R.S.; Sheehan, K.B.; Stout, R.G.; Rodriguez, R.J.; Henson, J.M. 2002. Thermotolerance generated by plant/fungal symbiosis. Science 298: 1581. DOI: 10.1126/science. 1078055

Spatafora, J.W.; Sung, G.H.; Sung, J.M.; Hywel-Jones, N.L.; White Jr., J.F. 2007. Phylogenetic evidence for an animal pathogen origin of ergot and the grass endophytes. Molecular Ecology 16: 1701-1711.

Tamura, K.; Peterson, D.; Peterson, N.; Stetcher, G.; Nei, M.; Kumar, S. 2011. MEGA5: Molecular evolutionary genetics analysis using maximum likelihood, evolutionary distance, and maximum parsimony methods. Molecular Biology and Evolution 28: 2731-2739.

Teixeira, M.A.; Bettiol, W.; Cesnik, R. 2001. Pathogenicity of Colletotrichum gloeosporioides, Orthezia praelonga pathogenicity agent, to citrus leaves, flowers and fruits $=$ Patogenicidade de Colletotrichum gloeosporioides, agente de patogenicidade da Orthezia praelonga, para folhas, flores e frutos cítricos. Summa Phytopathologica 27: 352-357 (in Portuguese).

Teixeira, M.A.; Bettiol, W.; Cesnik, R.; Vieira, R.F. 2004. Pathogenicity of the fungus Colletotrichum gloeosporioides, pathogen of Orthezia praelonga, to several fruits and to pumpkin seedlings. Revista Brasileira de Fruticultura 26: 356358 (in Portuguese, with abstract in English).
Thiers, B. 2018. Index Herbariorum: a global directory of public herbaria and associated staff. New York Botanical Garden's Virtual Herbarium. Available at: http://sweetgum.nybg.org/ science/ih/ [Accessed Nov 7, 2018]

van der Aa, H.A. 1978. A leaf spot of Nymphaea alba in the Netherlands. Netherlands Journal of Plant Pathology 84: 109115.

Vega, F.E. 2008. Insect pathology and fungal endophytes. Journal of Invertebrate Pathology 98: 277-279. DOI: 10.1016/j. jip.2008.01.008

Vega, F.E.; Posada, F.; Catherine Aime, M.; Pava-Ripoll, M.; Infante, F.; Rehner, S.A. 2008. Entomopathogenic fungal endophytes. Biological Control 46: 72-82. DOI: 10.1016/j. biocontrol.2008.01.008

White, T.J.; Bruns, T.; Lee, S.; Taylor. J. 1990. Amplification and direct sequencing of fungal ribosomal RNA genes for phylogenetics. p. 315-322. In: Innis, M.A.; Gelfand, D.H.; Sninksy, J.J.; White, T.J., eds. PCR protocols: a guide to methods and applications. Academic Press, San Diego, CA, USA. 